\title{
TEST MEASUREMENTS OF MODERNIZED VERSION OF TWO-DETECTOR DOPPLER SPECTROMETER
}

\author{
A. BARANowski ${ }^{a}$, M. Kostrzewa ${ }^{b}$ AND M. SzUSzKIEWICZ ${ }^{a}$ \\ ${ }^{a}$ Institute of Experimental Physics, Wrocław University \\ Pl. Maxa Borna 9, 50-204 Wrocław, Poland \\ ${ }^{b}$ Physical Laboratory, Technical University, Ozimska 75, 45-370 Opole, Poland
}

Basing on the electronic analogue modules in adding and subtracting branches of pulses, a modernized two-detector Doppler spectrometer has been constructed using only one multichannel analyser. Doppler spectra for $\mathrm{Al}, \mathrm{Ni}, \mathrm{Ag}$ have been measured. ACARFIT computer program was used to compare the Doppler and angular correlation of annihilation radiation results.

PACS numbers: $78.70 . \mathrm{Bj}, 07.85 .-\mathrm{m}$

\section{Introduction}

The quality of the annihilation quanta energy measurements (Doppler effect) has greatly increased when experimenters have begun to introduce Doppler apparatus modifications. In the basic version, the experimental set-up consisted of high energy resolution $\mathrm{Ge}(\mathrm{Li})$ detector, amplifier and multichannel analyser. A simple construction and short time of data acquisition were the main advantages of that version of the apparatus. Competitive, angular correlation of annihilation radiation (ACAR) method was better from the resolution point of view and not worse when considering the range of momentum distribution, but the ACAR method required high activity positron sources and data acquisition time was at least one order of magnitude longer than in Doppler measurements.

The first important modification of Doppler apparatus was connected with an increase in the measured momentum distribution range. It was possible by introducing a coincidence mode, where the second detector was used as the register of the second annihilation photon. The coincidence system considerably eliminated pile-up events, cosmic rays or, as in the case of using $\mathrm{Na}^{22}$ source, the Compton scattered nuclear photons $(1.28 \mathrm{MeV})$. It caused that the high momentum range of spectra, which had never been seen earlier, could be investigated [1-3]. The annihilation lines, measured in that convention, were asymmetrical due to the incomplete charge collection and it turned out to be a bit confusing because of the 
problems with background extraction. This problem was partly simplified by the analysis of only right parts of the annihilation lines.

The crucial moment of the Doppler apparatus evolution took place in 1975 when the authors of papers $[4,5]$ worked out a two-dimensional set-up. They used two high energy resolution $\mathrm{Ge}(\mathrm{Li})$ detectors in collinear geometry to register both annihilation quanta in the coincidence mode. The electric pulses were transformed into a numerical form to be recorded as pairs $\left(E_{1}\right.$ and $\left.E_{2}\right)$. The results of such measurements were usually presented in a two-dimensional coordinate system, where the dependence between the number of counts and energy difference $\Delta E$ includes an established range of the sum of both annihilation quanta. This way of the presentation of the results is analogous to the ACAR method. Proportionality of $\Delta E$ to the parallel momentum component is illustrated by the following formula:

$$
\Delta E=E_{2}-E_{1}=\frac{c \cdot p-p^{2} c^{2} / E_{\gamma}}{1-c \cdot p / E_{\gamma}} \cong c \cdot p .
$$

It is the same like the proportionality between angles and perpendicular momentum component in the ACAR method as long as momentum distribution is homogeneous. It is worth to say that thanks to the two-detector technique symmetrical Doppler spectra could be seen.

The two-detector Doppler spectrometer was always equipped with the developed selection part. The time and energy selection criteria, which could be precisely established, initiated a further progress in peak to background (PB) ratio and thus extended an approachable range of annihilating pair momentum distribution.

The next important advance in the two-dimensional set-up was connected with a better (by $\sqrt{2}$ ) than in previous versions relative apparatus resolution. The described improvements were obtained at the cost of time of data acquisition, which increased from a few hours to the order of three or four days. Nevertheless, the two-dimensional Doppler spectrometer seems to be competitive in relation to other methods.

\section{Experimental}

The technical details of the Doppler spectrometer will be published elsewhere [6], therefore in Fig. 1 only a general block diagram of the spectrometer is presented. The spectrometer consists of two high-energy resolution HPGe detectors, amplifiers, differential spectrum (the dependence between the number of counts and energy difference $\Delta E$ ) acquisition system or sum $\left(E_{T}\right)$ spectrum acquisition system with classical multichannel analyser. The triple selection mode enables us to limit the energies of the spectra independently and to limit the sum of the registered energies. Such selection equipment is very important because it influences the quality of $\mathrm{PB}$ ratio. The differential or sum acquisition system is simple in the solution and the numerical part of the system is not necessary. The time selection in our configuration depends mainly on the resolution time of the coincidence system and the other time adjustment elements.

In Fig. 2 the presentation of the differential spectrum for aluminium with full selection mode in the vicinity of the spectrum without selection and the spectrum without only sum selection is intentional, because this way evolution of the system can be followed. In the set-up without selection mode the differential peak can be 


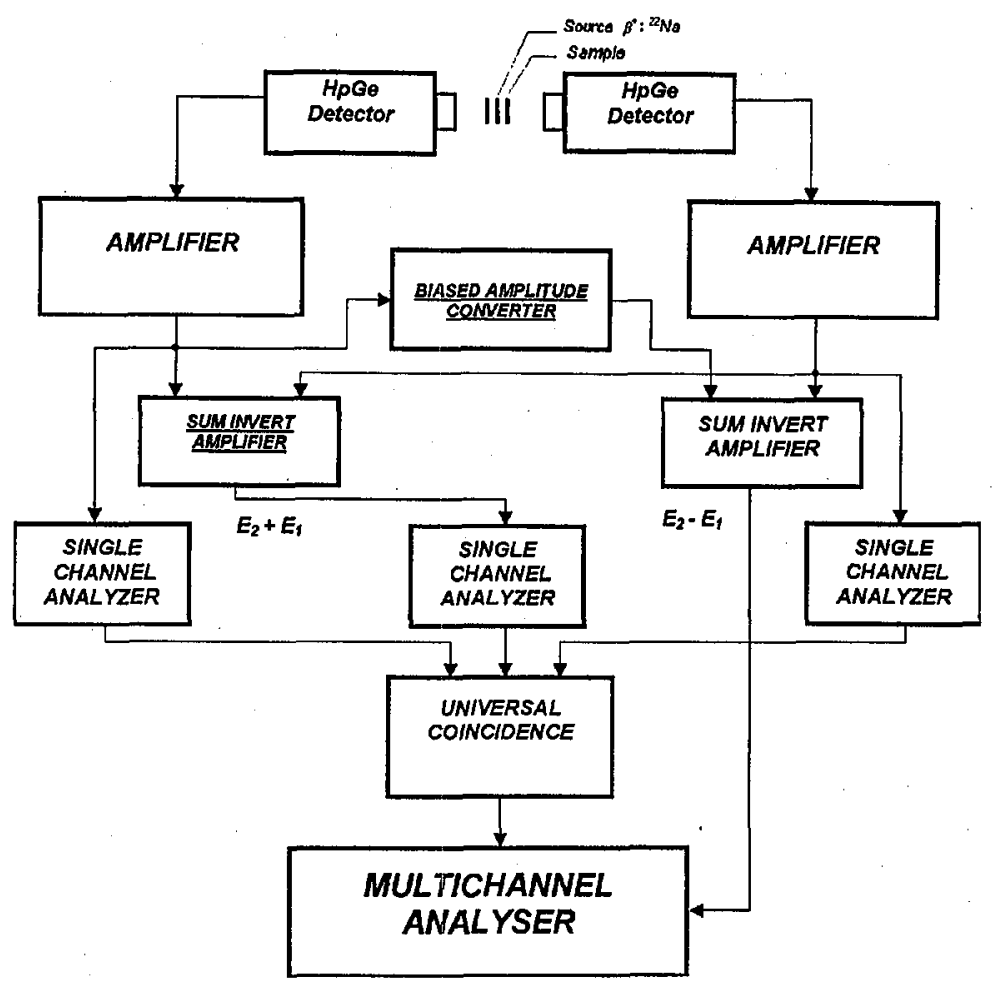

Fig. 1. General block diagram of two-detector Doppler spectrometer.

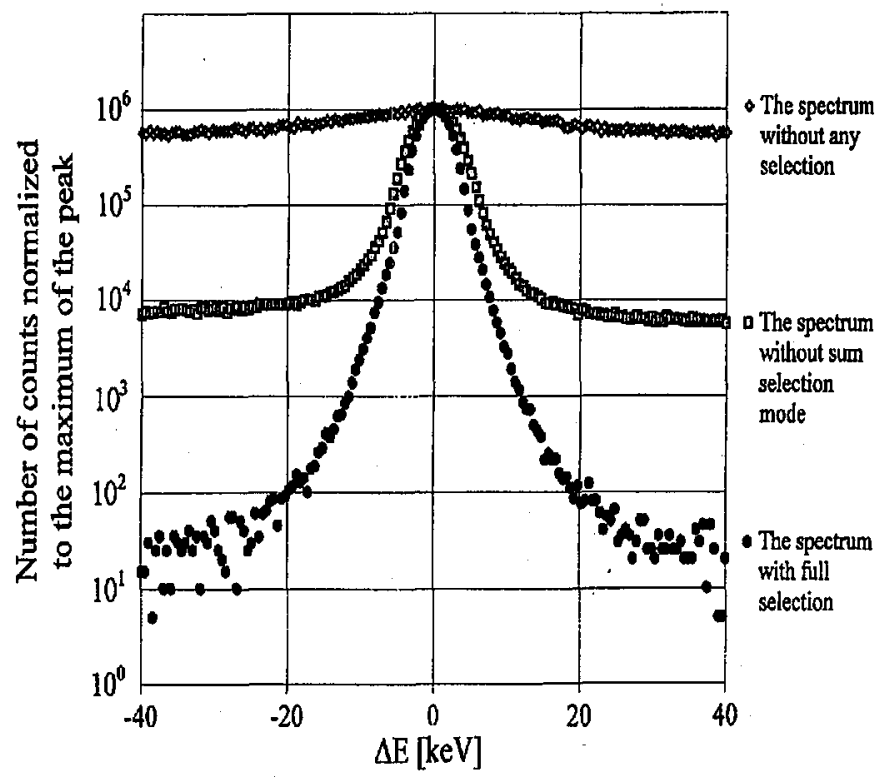

Fig. 2. The differential spectra for $\mathrm{Al}$ with three different selection modes. 
hardly seen due to the doubled (in comparison to the one-detector version) number of the registration of Compton scattered nuclear photons. It is well known that both detectors register high-energy photons without any restrictions. The small difference between the peak and the background is visually even diminished by semi-logarithmic scale. This way it is easy to see that the PB ratio achieves nearly five orders of magnitude.

The improvement, in comparison to the one-detector version, of relative resolution by $\sqrt{2}$ takes place when there are no additional elements in the acquisition line (detector, amplifier, multichannel analyser). Some modules as linear gates and delay amplifiers decrease apparatus resolution but practically high electronic technology and stable work conditions confined these factors to minimum. To calculate full width at the half maximum (FWHM) of the resolution curve the single line $\gamma$ source with an energy near $511 \mathrm{keV}$ is necessary, e.g., $\mathrm{Sr}^{85}$ with $514 \mathrm{keV}$. It is also possible to estimate the FWHM in a different way, using ACARFIT program [7]. Adaptation of angular correlation program to the Doppler necessities is well motivated by the analogy mentioned earlier in this article. The resolution curve in the ACARFIT program is described by one Gaussian and, which is important, can be counted automatically as the broadening of the parabola. There is another assumption that differential spectrum can be described by the composition of parabola and Gaussians. Finally estimated FWHM equals $2 \mathrm{keV}$, but taking into account that the differential apparatus measures the doubled effect $\left(E_{2}+\Delta E\right)-\left(E_{1}-\Delta E\right) \cong 2 \Delta E$, the relative resolution is even two times better, which means an improvement in comparison to the previous methods.

Transformation of the two-detector set-up into one-detector version with full selection is not complicated and can be done by disconnection of $E_{1}$ signal. In this version the $\mathrm{PB}$ ratio is the same like in two-detector. The only difference is connected with an increase in FWHM of the resolution curve (by a factor of about 1.3). This set-up can be recommended in the cases when two detectors differ considerably but even in that case a precise selection is necessary.

\section{Results}

High purity $(5 \mathrm{~N}) \mathrm{Ni}, \mathrm{Al}$, and $\mathrm{Ag}$ samples were chosen to test the apparatus. Each of the samples was annealed during 3 hours, in $75 \%$ of the melting temperature $[\mathrm{K}]$, and then slowly cooled down. After that, the results of lifetime measurements indicated only one component with typical values of lifetimes (155 ps for $\mathrm{Al}, 103 \mathrm{ps}$ for $\mathrm{Ni}$ and $127 \mathrm{ps}$ for $\mathrm{Ag}$ ). The aluminium and nickel, but not the same samples, were measured by the ACAR method. The ACARFIT computer program [7] was not used to attribute the physical meaning to the components but to compare the parameters of differential curves, differential curves after symmetrization and angular correlation curves. The results of ACARFIT analysis are presented in Table.

The comparison between experimental momentum distribution and rare gas core theoretical distribution calculated by Daniuk [8] for Ag and Ni samples has been done. On the picture (Fig. 3) we can see that both curves (theoretical and experimental) in the high momentum part of the spectra fit in quite well. It certifies the correctness of high momentum experimental distributions. 
TABLE I

The results of ACARFIT computer program analysis made for $\mathrm{Al}$.

\begin{tabular}{|c|c|c|c|c|}
\hline \multicolumn{5}{|c|}{ ALUMINIUM } \\
\hline & $\begin{array}{l}\text { Differential } \\
\text { spectrum }\end{array}$ & $\begin{array}{c}\text { Symmetrized } \\
\text { differential } \\
\text { spectrum }\end{array}$ & $\begin{array}{c}\text { Two-Component } \\
\text { decomposition } \\
\text { of the difference } \\
\text { spectrum }\end{array}$ & $\begin{array}{c}\text { Average } \\
\text { parameters from } \\
\text { the } 7 \text { angular } \\
\text { correlation curves }\end{array}$ \\
\hline \multicolumn{5}{|l|}{ PARABOLA } \\
\hline Cut-off [mrad] & $6.87(0.01)^{*}$ & $6.87(0.01)$ & $6.84(0.01)$ & $6.84(0.01)^{* *}$ \\
\hline Broadening [mrad] & $3.86(0.02)$ & $3.86(0.02)$ & $4.46(0.01)$ & \\
\hline Intensity [\%] & $73.2(0.3)$ & $73.2(0.3)$ & $82.3(0.1)$ & $82.2(0.3)$ \\
\hline GAUSSIAN 1 & & & & \\
\hline FWHM [mrad] & $13.9(0.1)$ & $13.9(0.1)$ & $16.45(0.02)$ & $14.69(0.01)$ \\
\hline Intensity [\%] & $24.0(0.2)$ & $24.1(0.2)$ & $17.7(0.1)$ & $17.8(0.3)$ \\
\hline \multicolumn{5}{|l|}{ GAUSSIAN 2} \\
\hline FWHM [mrad] & $24.3(0.3)$ & $24.6(0.3)$ & - & - \\
\hline Intensity [\%] & $2.7(0.1)$ & $2.6(0.1)$ & & \\
\hline \multicolumn{5}{|l|}{ GAUSSIAN 3} \\
\hline FWHM [mrad] & $67(2)$ & $72(2)$ & - & - \\
\hline Intensity [\%] & $0.1(0.01)$ & $0.1(0.01)$ & & \\
\hline Variance of the fit & $2.98(0.06)$ & $0.74(0.06)$ & $21.8(0.1)$ & $1.6(0.1)$ \\
\hline \multicolumn{5}{|c|}{ ALUMINIUM (One-Detector annihilation line with full selection) } \\
\hline & $\begin{array}{c}\text { Differential } \\
\text { spectrum }\end{array}$ & $\begin{array}{c}\text { Symmetrized } \\
\text { differential } \\
\text { spectrum } \\
\end{array}$ & Annihilation line & $\begin{array}{c}\text { Symmetrized } \\
\text { annihilation line }\end{array}$ \\
\hline \multicolumn{5}{|l|}{ PARABOLA } \\
\hline Cut-off [mrad] & $6.87(0.01)$ & $6.87(0.01)$ & $6.87(0.01)$ & $6.88(0.01)$ \\
\hline Broadening [mrad] & $3.86(0.07)$ & $3.86(0.02)$ & $5.17(0.02)$ & $5.17(0.03)$ \\
\hline Intensity [\%] & $73.2(0.3)$ & $73.2(0.3)$ & $77.8(0.4)$ & $77.8(0.4)$ \\
\hline \multicolumn{5}{|l|}{ GAUSSIAN 1} \\
\hline FWHM [mrad] & $13.9(0.1)$ & $13.9(0.1)$ & $14.9(0.2)$ & $15.0(0.1)$ \\
\hline Intensity [\%] & $24.0(0.2)$ & $24.1(0.2)$ & $18.8(0.3)$ & $19.1(0.4)$ \\
\hline \multicolumn{5}{|l|}{ GAUSSIAN 2} \\
\hline FWHM [mrad] & $24.3(0.3)$ & $24.6(0.3)$ & $24.3(0.4)$ & $24.7(0.4)$ \\
\hline Intensity [\%] & $2.7(0.1)$ & $2.6(0.1)$ & $3.2(0.3)$ & $3.0(0.2)$ \\
\hline \multicolumn{5}{|l|}{ GAUSSIAN 3} \\
\hline FWHM [mrad] & $67(2)$ & $72(2)$ & $77(2)$ & $83(2)$ \\
\hline Intensity [\%] & $0.1(0.01)$ & $0.1(0.01)$ & $0.1(0.01)$ & $0.097(0.004)$ \\
\hline Variance of the fit & $2.98(0.06)$ & $0.74(0.06)$ & $3.71(0.07)$ & $0.76(0.07)$ \\
\hline
\end{tabular}

"Statistical errors are placed in round brackets. ${ }^{* *}$ Arithmetical statistical error.

The construction of the one-detector apparatus, working with full selection or without selection, creates an occasion to prove some numerical methods of background extracting. With regard to the high value of $\mathrm{PB}$ ratio, the background in the full selection curves can be nearly neglected. Therefore, the annihilation 


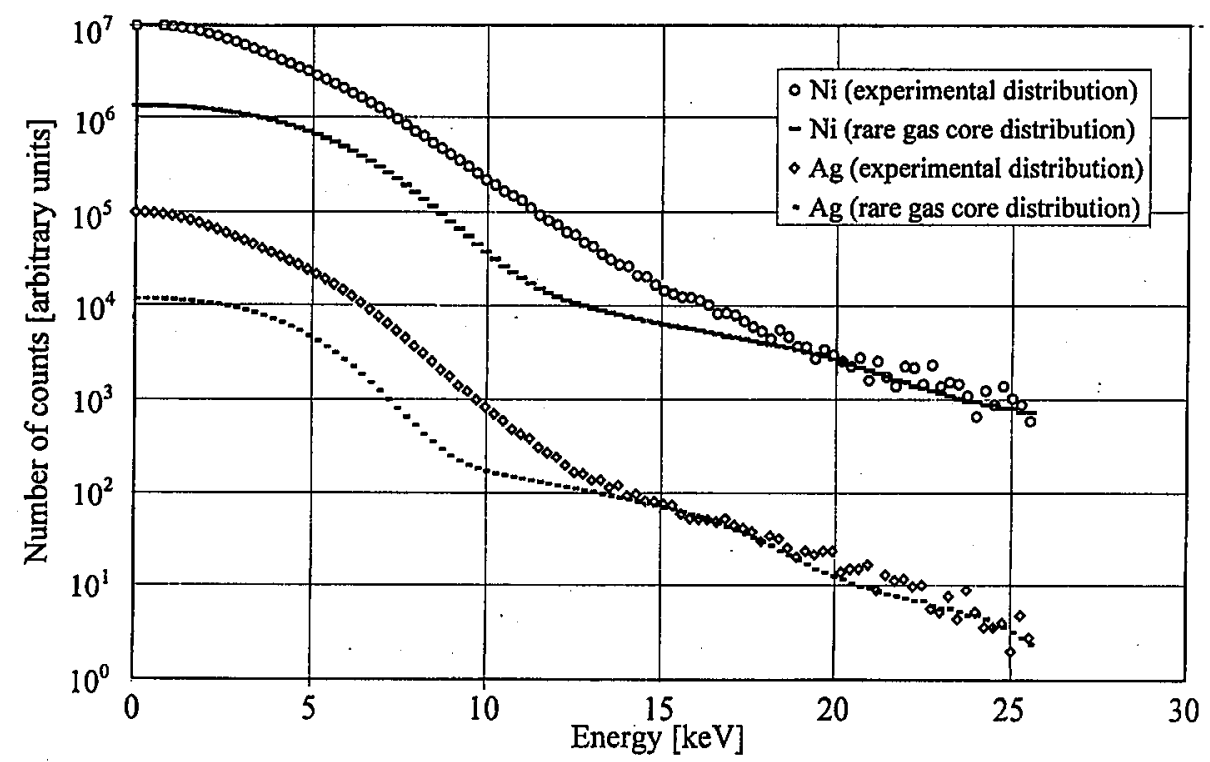

Fig. 3. The comparison between theoretical and experimental rare gas core distribution made for $\mathrm{Ni}$ and $\mathrm{Ag}$.

lines (without selection) after numerical background extraction should be similar to the ones fully experimental. Gunnink and Wätzing [9-11] proposed one of the most suitable functions describing the background

$$
B G=n_{k_{r}}-\frac{\sum_{i=k}^{k_{r}} n_{i}}{\sum_{i=k_{l}}^{k_{r}} n_{i}}\left(n_{k_{r}}-n_{k_{l}}\right)
$$

where $k$ is the channel number, $n_{i}$ - the number of counts in the $i$-th channel, $k_{l}$ and $k_{r}$ are the channels far away from the centre of the peak on the left and right sides, where only the background exists. The next function suggested by Helmer and Lee [11] corrects the tail part of the spectrum

$$
T(k)=p_{1} \exp \left(-p_{2}\left|k-k_{\mathrm{c}}\right|\right)\left[1-\exp \left(-p_{3}\left(k-k_{\mathrm{c}}\right)^{2} / 2 \sigma^{2}\right)\right],
$$

where $p_{1,2,3}$ are unphysical parameters but characterize the special Ge detector used for the measurements, $\sigma$ is the standard deviation of the Gaussian representing the main part of the peak, $k_{\mathrm{c}}$ is the channel where the centre of the peak is located. The results of the curve modifications are presented on the sequence of the pictures. It can be seen in Figs. 4 and 5 that after only two steps of the analysis very good agreement between both curves is observed. It certifies the confidence of both methods. 

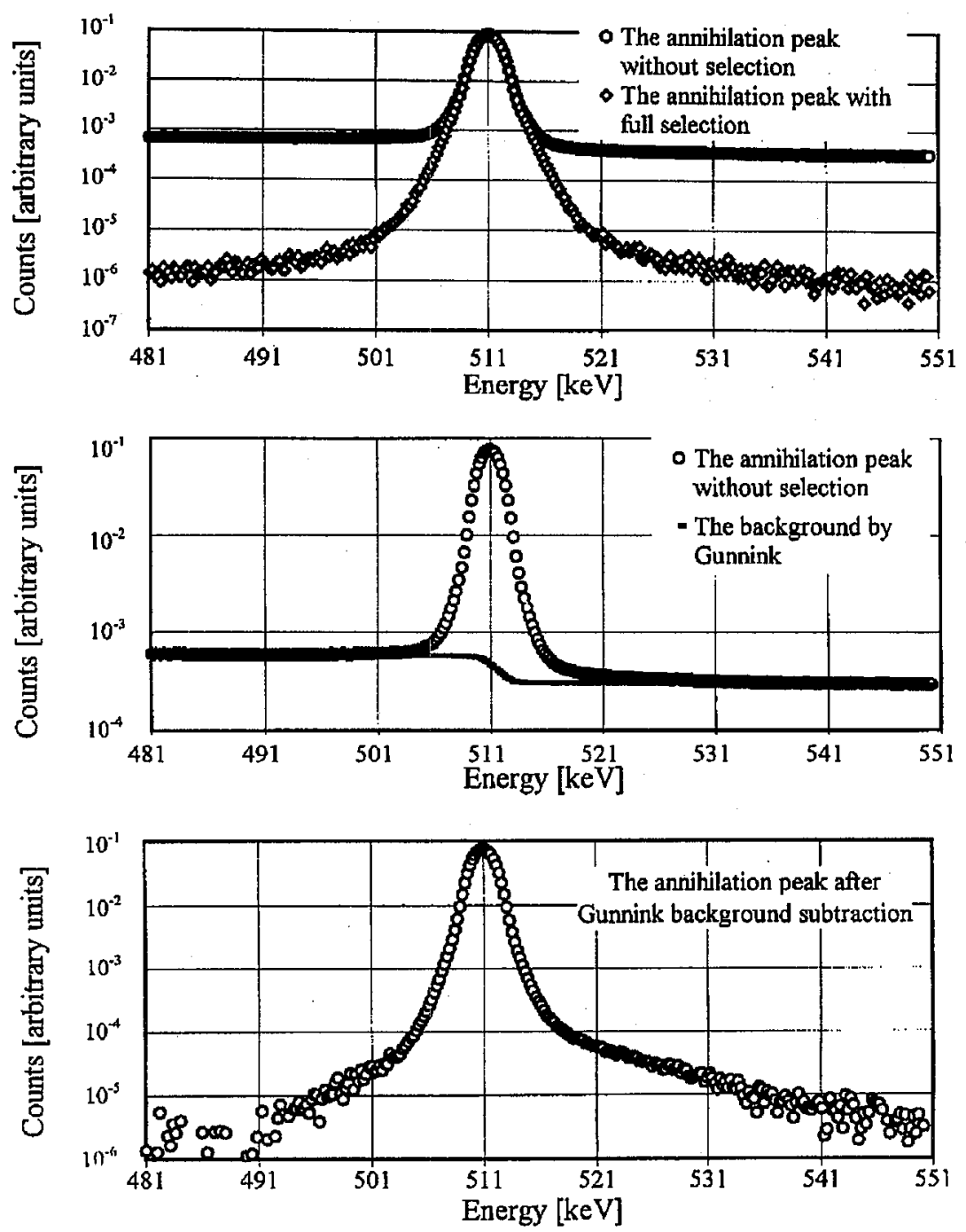

Fig. 4. The first step of the background extraction.

\section{Conclusions}

The peak to background ratio of the typical spectrum (using $\mathrm{Na}^{22}$ positron source) is approximately equal to $5 \times 10^{4}$ and the relative apparatus resolution is about $30 \%$ better compared to a conventional one-detector equipment. The results certify reliability of modernised version of Doppler apparatus. ACARFIT analysis pointed out that:

- symmetrization improves only variance of the fit because the results practically do not differ,

- there is consistency between parameters describing differential curve and annihilation curve (stored by one-detector version of Doppler apparatus with full selection), 

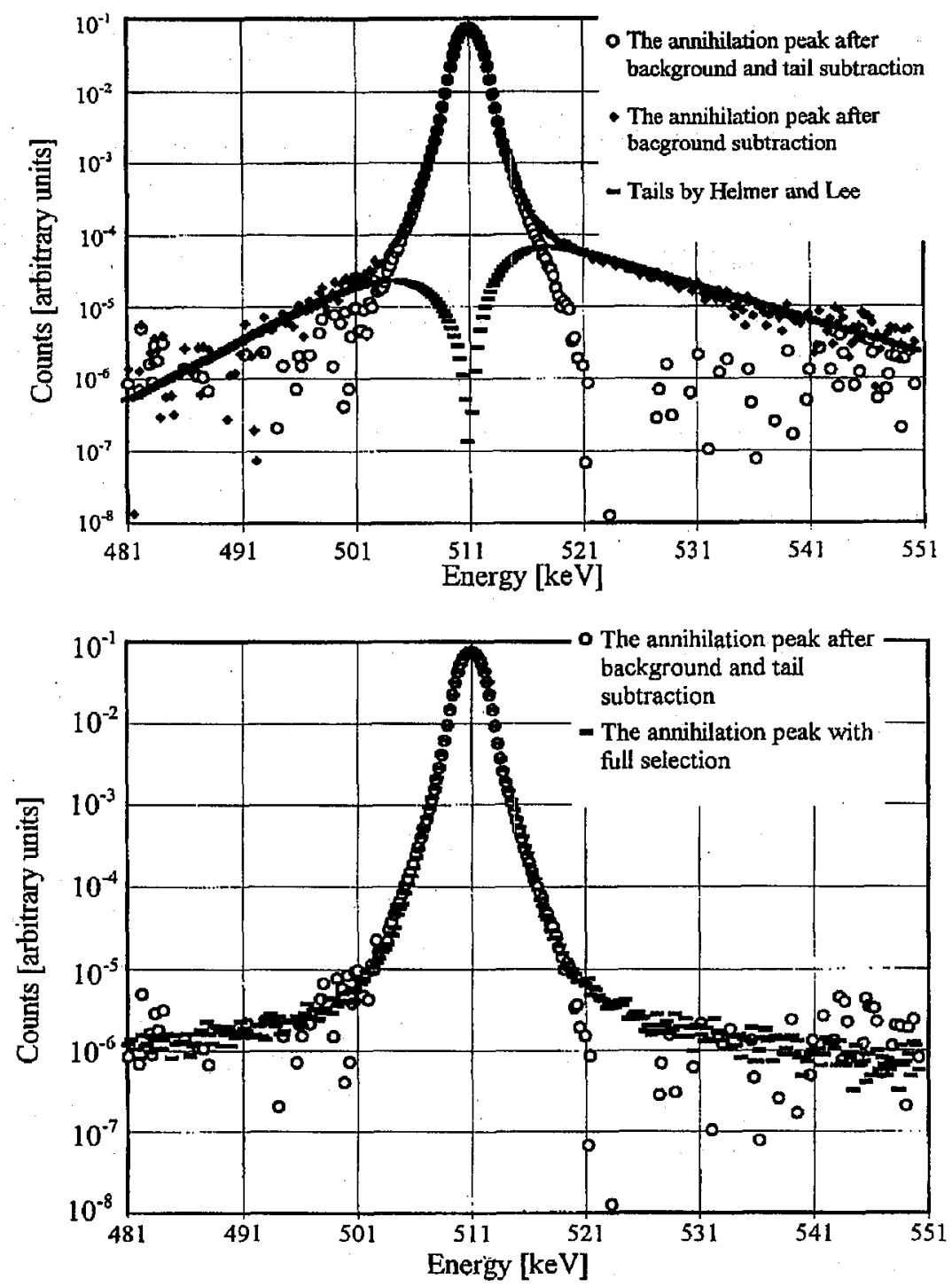

Fig. 5. The second step of the background extraction.

- the greatest differences can be found between angular correlation spectrum and differential spectrum but it should be stressed that the disparity is still rather small and can be mostly explained by comparison methodology. The momentum distribution ranges of both methods considerably differ and as expected the same results must not be really the same.

This work was supported by the University of Wroctaw under the grant 2016/IFD/98. 


\section{References}

[1] K.G. Lynn, A.N. Goland, Solid State Commun. 18, 1549 (1976).

[2] G. Pruban, M.Sc. Thesis, Wrocław University, Wrocław 1994.

[3] J. Dryzek, C.A. Quarles, Nucl. Instrum. Methods A 378, 337 (1996).

[4] J.R. MacDonald; R.A. Boie, L.C. Feldman, M.F. Robbins, P. Mauger, K.G. Lynn, Bull. Am. Phys. Soc. 24, 580 (1975).

[5] J.R. MacDonald, K.G. Lynn, R.A. Boie, M.F. Robbins, Nucl. Instrum. Methods 153, 189 (1978).

[6] A. Baranowski, M. Kostrzewa, M. Szuszkiewicz, prepared to publish in Nucl. Instrum. Methods.

[7] P. Kirkegaard, N.J. Pedersen, M. Eldrup, Grafisk Service, Risø-M-2740 (1989), p. 16.

[8] S. Daniuk, private information.

[9] R. Gunnink, in: Proc. Am. Nucl. Soc. Topical Conf. on Computer in Activation Analysis and Gamma-Ray Spectroscopy, Mayaguez (Puerto Rico) 1978, CONF-780421, 1979, p. 109.

[10] W. Wätzing, R. Gunnink, in: Proc. Am. Nucl. Soc. Topical Conf. on Computer in Activation Analysis and Gamma-Ray Spectroscopy, Mayaguez (Puerto Rico) 1978, CONF-780421, 1979, p. 150.

[11] R.G. Helmer, M.A. Lee, Nucl. Instrum. Methods 178, 499 (1980). 